\title{
Otosclerosis Revisited
}

\author{
C V Srinivas ${ }^{1}$
}

\begin{abstract}
Introduction:
ABSTRACT

Otosclerosis usually manifests as a progressive conductive or mixed hearing loss occurring clinically to varying degrees in $0.5 \%-1 \%$ of the general population. Stapedectomy /stapedotomy is the current treatment of choice for conductive component of Otosclerosis.

Materials and Methods:

Sixty patients attending the department of ENT of a Medical College Hospital in Bangalore between October 2012 and October 2017 were included in the study.

Results:

The incidence of otosclerosis is highest in the $3 \mathrm{rd}$ and 4 th decade. Bilateral symptoms were present in $25 \%$ of the patients and $75 \%$ had unilateral symptoms, right side was more common in our study. Tinnitus and vertigo with deafness were seen in $60 \%$ and $25 \%$ of the patients respectively.

Ninety percent of the patients presented with moderate to moderately severe hearing loss. Twenty four (40\%) out of 60 audiograms studied showed Carhart's notch. Post-operative closure of air-bone gap to within 10dB could be achieved in $65 \%$ cases. The $p$ value calculated comparing pre and post op results is $<0.0001$ and significant.

Vertigo was found to be most common post-operative complication (35\%) followed by sensorineural hearing loss (6.6\%). Other complications observed during the study include - tympanic membrane tears, foot plate extrusion, lax long process of incus, perilymph leak.

\section{Conclusion:}

From the assessment of the postoperative hearing gain and postoperative complications, it can be concluded that small fenestra stapedotomy gives satisfactory post-operative outcome and may be appropriatein the Indian scenario.

Keywords:

Otosclerosis; Stapedotomy
\end{abstract}

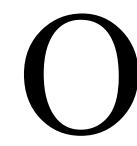
tosclerosis is one of the commonest non infective causes of acquired deafness in adults'. It is a primary and exclusive disease of otic capsule of human temporal bone. Otosclerosis is characterized by alternate phases of bone resorption and formation. If the location of bony changes produces evident clinical manifestations the term "Clinical Otosclerosis" is used. If bonychangesarenottranslatedintoclinicalmanifestations, the term used is "histological Otosclerosis". Antonio Valsalva in 1735 gave the first description of ankylosis of stapes to margins of oval window. Von Troltsch in 1881 coined the term 'Otosclerosis'. Politzer in 1893 first described Otosclerosis as a primary disease of otic

1 - Department of ENT, Dr B R Ahmed Medical College and Hospital, Bangalore

Corresponding author:

Dr C V Srinivas

email: shruthi_dechu@yahoo.co.in capsule. Otosclerosis usually manifests as a progressive conductive or mixed hearing loss occurring clinically to varying degrees in $0.5 \%-1 \%$ of the general population. Therapeutic options for Otosclerosis include medical, surgical and use of hearing aids, alone or in combination. Stapes surgery is an effective treatment for hearing loss and tinnitus of Otosclerosis and stapedectomy/ stapedotomy is the current treatment of choice for conductive component of Otosclerosis.

The procedure of extracting the stapes for Otosclerosis was first performed by Jack of Boston in 1892 but was beset with obvious difficulties because he lacked proper magnification and antibiotic coverage. In 1956 Dr. John Shea revived the stapedectomy operation for Otosclerosis and replaced the stapes bone with a polyethylene tube prosthesis and vein graft. Stapedotomy is a more precise method of creating a hole in footplate rather than total footplate removal. It gives less post-operative vertigo 
and better high frequency hearing compared to total footplate removal.

The present study is a longitudinal clinical study on Otosclerosis with emphasis on clinical and audiological features, per operative findings, outcome and complications of stapedectomy. Patients with stapedial otosclerosis, above 12 years of age of both gender were included in the study, however patients with pure sensorineural hearing loss were excluded from the study. The objective is to study the demographic and clinical features of Otosclerosis; to analyze the results of audiometry and operative notes of Otosclerosis; to describe the outcome and incidence of complications of stapes surgery in Otosclerosis.

\section{Materials and Methods}

The study included 60 patients who were diagnosed to have clinical Stapedial Otosclerosis. They underwent small fenestra stapedectomy in the Department of ENT of a Medical College Hospital in Bangalore between October 2012 and October 2017. Thus totally 60 ears affected by Otosclerosis were studied.

Clinical Evaluation: After recording demographic data, a thorough history was taken and clinical examination performed. The onset, duration, progression of hearing loss was recorded. History of tinnitus elicited. Otoscopic examination and Tuning Fork tests were performed. Tuning fork tests with 256, 512 and $1024 \mathrm{~Hz}$ are done. Haematological investigations along with investigation for Cardiovascular and Respiratory System were done. An informed consent was taken explaining all the possible complications associated with the surgery. Patients were kept nil per mouth overnight. A day before thorough shampoo and head bath was advised to all the patients. Premedication was given by Inj Pentazocine $30 \mathrm{mg}$ and Promethazine $25 \mathrm{mg}$ via intramuscular route.

Technique of Small Fenestra Stapedectomy: Local infiltration of the External Auditory Meatus is done with $2 \%$ lignocaine with 1:2,00,000 adrenaline. All the patients are operated upon by the author.

Vascular strip is carefully injected with $2 \%$ lignocaine with adrenaline (1:200000). A tympanomeatal flap is raised, the chorda tympani nerve is identified and preserved to gain exposure to the oval window region. Tip of the pyramidal process and the horizontal portion of facial canal over the oval window are visualized. The Stapedial tendon is divided sharply. The stapes superstructure is removed. A small (approximately $0.6 \mathrm{~mm}$ in diameter) fenestra is made in the posterior half of the footplate using a pick or hand drill. The Teflon piston $(0.5 \mathrm{~mm}$ diameter $)$ is then inserted through the fenestra to a depth of about $0.25 \mathrm{~mm}$ in the vestibule. It is then crimped to the long process of Incus. A tissue seal is made around the piston with fat, blood clot or gel foam.

Post-operative Care: Post operatively patients were continued on broad spectrum antibiotics with analgesic, antihistamine and systemic decongestant given to avoid nasal discharge and upper respiratory tract infection. Patients without any complications were discharged on second post-operative day. Patients were advised to avoid sneezing and sudden movement of the head and neck for 48 hours. On discharge, oral form of antibiotic was given with antihistamine for next one week.

Follow up: Patients were advised to attend the outpatient department on sixth post-operative day and ear was examined. Antibiotic continued for 3 weeks. Antihistamine was continued for another two weeks. During the third post-operative visit at 4 weeks, the gel foam, if remaining, was removed and status of tympanic membrane checked. External ear canal and tympanic membrane is especially looked for any granulation tissue formation. Any other complaints if present are tackled accordingly. Patients were advised to come after three months. During this visit status of tympanic membrane is again assessed by otoscopy. Pure tone audiogram is done and the results compared with pre-operative audiogram. Regarding ethical point of view no patients are subjected to any further risk during the study. No further investigations are done. No new methods were tried during this study.

\section{Results}

The age of patients varied from 20-50 yrs. Majority of patients were in 30-40 years age group (56.6\%). The next common age group is 21-30 years. Age and gender distribution and age of onset of hearing loss is shown in 
Table I: Age and gender distribution, age of onset of symptoms

\begin{tabular}{|c|c|c|}
\hline GENDER & NUMBER & PERCENTAGE \% \\
\hline Male & 25 & 52 \\
\hline Female & 35 & 5 \\
\hline AGE OF ONSET & NUMBER & PERCENTAGE \% \\
\hline$<10$ & 3 & 20 \\
\hline $11-20$ & 12 & 40 \\
\hline $41-40$ & 15 & 10 \\
\hline
\end{tabular}

Table II: The side of involvement and incidence of symptoms

\begin{tabular}{|c|c|c|}
\hline SIDE AFFECTED & NUMBER & PERCENTAGE \% \\
\hline Both sides & 15 & 25 \\
\hline Predominantly Right & 24 & $\mathbf{3 0}$ \\
\hline Predominantly Left & 21 & $\mathbf{1 0 0}$ \\
\hline SYMPTOMS & NUMBER & PERCENTAGE \% \\
\hline Deafness & 60 & $\mathbf{6 0}$ \\
\hline Deafness+tinnitus & 36 & $\mathbf{2 5}$ \\
\hline Deafness+ vertigo & 15 & 15 \\
\hline Paracusis & 9 & 2 \\
\hline
\end{tabular}


Table I.

Patients below the age of 20 years were diagnosed clinically and classic Carhart's notch.

Twenty five percent patients presented with bilateral symptoms and $75 \%$ had unilateral symptoms. Hearing loss was present in all the patients. Other associated symptoms were tinnitus and vertigo, shown in Table II.

Patients gave history of vertigo with deafness were included in the study, however they were operated when they did not have an active episode of vertigo.

Tympanic membrane: Otoscopy of the patients in the present study showed normal appearance in 45 patients(75\%), retraction in 9 patients $(15 \%)$ and myringosclerosis in 6 patients $(10 \%)$. No middle ear pathology was found in these patients after exploratory tympanotomy.

Approximately $90 \%$ of the patients presented with moderate to moderately severe hearing loss. There are no patients with minimal or profound hearing loss. 24 (40\%) out of 60 audiograms studied showed Carhart's notch $.90 \%$ of the patients showed air-bone gap between $31-50 \mathrm{~dB}$. The study showed 'As' type of curve in (27) $45 \%$ of the patients.

The details of the audiological investigations are shown in Table III.

Patients with mild conductive hearing loss (21-30 dB) also were surgically treated as per Robert Vincet, Causse's Clinic protocol was followed in the current study.

No middle ear pathology found intraoperatively in patients with B type tympanogram.

Length of the piston used in present study: Piston length of $5 \mathrm{~mm}$ is used in $60 \%$ of cases and $4.5 \mathrm{~mm}$ in $20 \%$ of cases , $4.75 \mathrm{~mm}$ in $15 \%$ cases, $4.25 \mathrm{~mm}$ in $5 \%$ cases.

Complications following surgery: During the post operative period $35 \%$ of the patients presented with the complaints of vertigo, which lasted for three to five days and treated conservatively. 6.6\% had SNHL postoperatively. (Table IV)

Tympanomeatal tear was found in 15 patients was repaired using tragal perichondrium.

\section{Discussion}

In the present study 60 patients underwent small fenestra stapedectomy for Stapedial Otosclerosis, their demographic data, clinical findings, audiological assessment are recorded and analyzed. Complications following small fenestra stapedectomy are also analyzed. Although the sample size was small, the results obtained are compared to similar studies in the literature. The mean age of patients included in the study is 34 years. Mean age of male patients is 36 and female patients is 33years. In the present study more younger female patients are found with Otosclerosis than male patients. In 2005 Quaranta et al in a study of 151 patients with Otosclerosis, found the mean age to be 46 years. ${ }^{1}$ The mean age of onset of symptoms in this study is 28 years. In a similar study the mean age of onset in different countries is found to be; 33 in Panama, 29 in Minnesota, 24 in Chile and 23 in Brazil.

In the present study the female preponderance is 1:2 which is similar to other studies by Schmidt in 1933 (72.5\%), by Shambaugh in $1952(68 \%)$ and by Cawthorne in $1955(67 \%){ }^{2}$ Comparison of studies for bilateral disease showed a large incidence in the present study (83.7\%); in a study by Glasscock et al the incidence was $72 \%$, Ginsberg et al had $80 \%$ and in a study by Levy et al it was $66 \%$. The primary symptom in Otosclerosis is gradually progressive hearing loss. The magnitude of hearing loss is directly related to the degree of fixation of stapes footplate and the duration of hearing loss. $90 \%$ of the patients presented within 3 yrs of onset of symptoms. The duration of hearing loss was studied by Lippy et $\mathrm{al}^{3}$ in 1999 compared the trend of disease in the 1960's, 1970's, 1980's and 1990's and found that incidence to decrease from 18.3 years in 1960 's to 14.6 years in 1970's, 16.3 years in 1980's and 11.1 years in 1990 's; $60 \%$ of the patients in this study gave history of Tinnitus.

History of vertigo is elicited in $25 \%$ of the patients. Goodhill proposed that tinnitus in Otosclerosis was a class of 'unmasked visceral tinnitus' which could be a feature of any pathology associated with conductive deafness and is due to normally sub audible tympanic and peritympanic vascular and muscular noises that are unmasked by the conductive deficit. ${ }^{4} \mathrm{~A}$ positive history 
Table III: Showing pre-op audiological findings and post operative a-b gap

\begin{tabular}{|c|c|c|}
\hline PRE-OP HEARING LOSS & NUMBER & PERCENTAGE \% \\
\hline Minimal (15-25) & $\mathbf{0}$ & $\mathbf{0}$ \\
\hline Mild (16-40) & 6 & 10 \\
\hline Moderate (41-55) & 21 & 35 \\
\hline Moderately severe (56-70) & 27 & 45 \\
\hline Severe (71-90) & 6 & 10 \\
\hline Profound (>90) & $\mathbf{0}$ & $\mathbf{0}$ \\
\hline PRE-OP A-B GAP & NUMBER & PERCENTAGE \% \\
\hline 0-10 & $\mathbf{0}$ & $\mathbf{0}$ \\
\hline 11-20 & $\mathbf{0}$ & $\mathbf{0}$ \\
\hline 21-30 & 3 & 5 \\
\hline 31-40 & 30 & 50 \\
\hline 41-50 & 24 & 40 \\
\hline$>\mathbf{5 0}$ & 3 & 5 \\
\hline PRE-OP TYMPANOGRAM-TYPE & NUMBER & PERCENTAGE \% \\
\hline $\mathbf{A}$ & 30 & 50 \\
\hline As & 27 & 45 \\
\hline B & 3 & 5 \\
\hline POST OP ABG (DB) & NUMBER & PERCENTAGE \% \\
\hline $0-5$ & 30 & 50 \\
\hline $06-10$ & 9 & 15 \\
\hline 11-15 & 15 & 25 \\
\hline $16-20$ & 6 & 10 \\
\hline$>\mathbf{2 0}$ & $\mathbf{0}$ & $\mathbf{0}$ \\
\hline
\end{tabular}


Table IV: Showing incidence of post operative complications

\begin{tabular}{|c|c|c|}
\hline COMPLICATIONS & NUMBER & PERCENTAGE \% \\
\hline Vertigo & 21 & 35 \\
\hline SNHL & 4 & 6.6 \\
\hline Revision piston displacement & 2 & 3.4 \\
\hline Footplate extrusion \& replacement & 1 & 1.6 \\
\hline Lax long process of incus & 1 & 1.6 \\
\hline Injury to chorda tympani & 0 & 0 \\
\hline Injury to facial nerve & 0 & 0 \\
\hline Perilymph leak & 1 & 1.6 \\
\hline Tympanic membrane tear & 15 & 25 \\
\hline A/C otitis media & 0 & 0 \\
\hline Labyrinthitis & 0 & 0 \\
\hline Meningitis & 0 & 0 \\
\hline
\end{tabular}

of Paracusis Willisii was found in $15 \%$ of patients. Wager in 1939 showed familial incidence in $58 \%$ of patients in his study. Cawthorne showed the incidence to be $54.5 \%$ and Larsson in $49 \%$ of his patients. Worsening of hearing is noted in $60 \%$ of his patients by Panama in 1987. Similarly clinical onset of Otosclerosis is reported in $16.6 \%$ of the patients of Brazil, $14 \%$ of Puerto Rico and $10 \%$ of Minnesota patients in his study.

The physical appearance of tympanic membrane is usually normal in most patients with Otosclerosis. In about $10 \%$ a positive Schwartz's sign is identifiable. In this study $75 \%$ patients had a normal intact tympanic membrane, $15 \%$ had mild retraction and $10 \%$ had myringosclerosis. The key objective measurement in Otosclerosis remains the pure tone audiogram. $45 \%$ of the patients had moderate to moderately severe hearing loss pre operatively and $90 \%$ had a-b gap of $31 \mathrm{~dB}$ to 50
$\mathrm{dB}$. Air bone gap below $20 \mathrm{~dB}$ and above $55 \mathrm{~dB}$ is not seen in the present study. Type A Tympanogram is found in $50 \%$ of the patients and As type of Tympanogram is seen in $45 \%$ of the patients. As the middle ear aeration is un-affected in Otosclerosis Type A Tympanogram is seen usually and As type of curve is seen in later stages. ${ }^{5}$ In the present study all the patients are operated through transcanal approach.

In a study published in 1999 by Lippy et al, a decrease in incidence of obliterative footplate was noted. Smyth and Hazzard (1978) in a study of 655 cases found an incidence of $64 \%$ in circumferential type, $28 \%$ in biscuit footplates and $8 \%$ in Obliterative Otosclerosis. ${ }^{6}$ Various types of pistons are preferred by different authors. In the present study Teflon piston of $0.5 \mathrm{~mm}$ diameter is used in all the patients. The length of the piston depends on the distance from the incus to the stapes footplate. This 
distance together with an extra $0.25 \mathrm{~mm}$ (extra length is the depth to be inserted into the vestibule) is measured in each case and the length of the piston was cut to size with measuring jig and rod. Most commonly used length was $5 \mathrm{~mm}(60 \%)$, followed by $4.5 \mathrm{~mm}(20 \%)$, $4.75 \mathrm{~mm}(15 \%)$ and $4.25 \mathrm{~mm}(5 \%)$. Post-operative Pure Tone Audiogram is taken at 3 months. Post-operative Air Bone Gap (AB gap) recorded in all the patients is analyzed. $65 \%$ of the patients showed $A B$ gap within $10 \mathrm{~dB}$ and the rest showed within $20 \mathrm{~dB}$. There are no patients with $\mathrm{AB}$ gap more than $20 \mathrm{~dB}$ in this study. The mean post-operative $\mathrm{AB}$ gap is $9.2 \mathrm{~dB}$ in this study. The Pre-Operative and Post-Operative results of closure of $a-b$ gap is statistically significant with $p$ value $<0.001$. By conventional criteria this difference is considered to be extremely statistically significant. Quaranta et al in 2005 reported $84.8 \%$ of small fenestra stapedectomy had a post-operative $\mathrm{AB}$ gap within $10 \mathrm{~dB}$ and mean post-operative $\mathrm{AB}$ gap as $6 \mathrm{~dB}$.

The most common post-operative complication which occurred in our study is post-operative vertigo $(35 \%)$. Tympanic membrane tear occurred in five cases $(25 \%)$. During Post -Operative follow up with audiogram, evidence of SNHL found in $6.6 \%$ the present study. Palva et al reported in his study of 456 ears (360 patients) of three drum perforations - one infected and two dry; perilymph fistula in 7 and sensorineural hearing loss in 6. ${ }^{7}$ Larsson et al reported delayed facial palsy in 7 out of 706 stapedectomy operations. They proposed a viral origin and suggested prophylactic therapy with acyclovir during the whole perioperative period in all patients with history of HSV reactivation. ${ }^{8}$ Hannley et al in 1993 reported a $2.8 \%$ rate of sensorineural hearing loss after stapedectomy. ${ }^{9}$ Lippy et al in 1999 encountered 2 cases of sensorineural hearing loss and 6 cases of tympanic membrane tear in their study. Surgical approaches in treating Otosclerosis need not be stereotyped. It is possible to use a variety of techniques, depending on the pathologic conditions and anatomic characteristics encountered during stapes surgery.

The major objective is to improve hearing significantly, with or without a hearing aid and to avoid complications. This is a limited study with 60 patients, insufficient to give a significant epidemiological record. The duration of the study and follow up period is also not sufficient to give long term effects of small fenestra Stapedectomy response. Stapes surgery is indicated in a patient with hearing threshold $20 \mathrm{~dB}$ or worse, a negative Rinne at three consecutive lower frequencies of 256, 512 and $1024 \mathrm{~Hz}$. The speech discrimination if found good is an added benefit of predicting favourable prognosis. ${ }^{10}$ Stapes should be firmly fixed and when there is very early partial fixation, stapes surgery is best delayed until fixation is more complete because there is only a little hearing improvement to be gained and much to be lost in case of severe cochlear reaction. ${ }^{11}$ A successful stapes surgery not only corrects the entire conductive component of loss but it also removes the variable Carhart's notch with over closure of the preoperative air-bone gap. Thus stapes surgery can be used for improving the hearing aid usage in presence of stapes fixation and a profound hearing loss, provided there is good speech discrimination.

\section{Conclusion}

Present study shows the incidence of Otosclerosis is similar to the literature reviewed world over; this study showed same incidence in both gender and the age group is between 3rd and 4th decades. All patients in our study presented with hearing loss (100\% cases). Small fenestra Stapedotomy is an ideal method of surgical intervention in the Indian Scenario as it is devoid of major post-operative complications or vertigo. $5 \mathrm{~mm}$ length piston was used in $60 \%$ cases with minimal post operative complications. Small fenestra Stapedotomy results showed mean closure of a-b gap to be $10 \mathrm{~dB}$ in the study which is significant statistically and may be appropriate in the Indian context.

\section{References}

1. Quaranta N, Besozzi G, Fallacara RA, Quaranta A. Air and bone conduction change after stapedotomy and partial stapedectomy for otosclerosis. Otolaryngol Head Neck Surg. 2005; 133:11620

2. GlasscockME, Shambaugh GE Ed. Clinical diagnosis of otosclerosis. In Surgery of the ear. 6th Ed. WB Saunders Co. 2010; 531-43

3. Lippy WH, Berenholz LP, Burkey JM. Otosclerosis in the 1960s, 1970s, 1980s, and 1990s. The Laryngoscope 2012; 146 
: 109-13

4. Goodhill V. The use of cortisone in otosclerosis. Transactions - American Academy of Ophthalmology and Otolaryngology. $192 ; 56: 635-46$

5. Cawthorne T. Otosclerosis; a review of its clinical features as noted in a consecutive series of 1150 cases. Acta otolaryngologica 1951; 40: 160-79

6. Smyth G. Otosclerosis. In Scott-Brown's Otolaryngology, Butterworth- Heinemann 6th ed. 1997. Vol. 3, 3/14/3-5.

7. Palva T, Karja J, Palva A. Otosclerosis surgery. Acta oto- laryngologica 1977; 83:328-35

8. Larsson A. Otosclerosis: A genetic and clinical study. Acta Otolaryngol suppl. 1960; 154:1-86

9. Hannley MT. Audiologic characteristics of Otosclerosis. Otolaryngologic clinics of North America 1993; 26:373-87

10. Causse JB. The twenty fine points of otosclerosis surgery. The American Journal of Otology 1989; 10:75-7

11. Hall IS. Surgical Treatment of Otosclerosis. Proceedings of the Royal Society of Medicine 1944; 37:737-43. 